DETC2008-49340

\title{
HOLISTIC ENGINEERING DESIGN: A COMBINED SYNCHRONOUS AND ASYNCHRONOUS APPROACH
}

\author{
A P Conway / University of Strathclyde
}

M D Giess / University of Bath

\author{
A Lynn / University of Strathclyde \\ L Ding / University of Bath \\ Y M Goh / University of Bath
}

\section{A McMahon / University of Bath}

the artifact being developed but also on the process through which this development occurs. Studies have shown that current documentary approaches are not sufficient to capture activities and decisions in their entirety [1-4], and can lead to organizations revisiting and in some cases reworking design decisions in order to understand previous design episodes.

In the past decade, there has been a significant amount of work focused on aiding the documentation of information within the product design lifecycle, as it is widely acknowledged that creating accurate representations of design activities can provide significant advantage to organizations later in the product lifecycle if they are properly documented.

However, design activities may be considered and classified along numerous dimensions, each with different modes. For example, it is possible to identify modes of learning and transaction to describe whether an activity is aimed at increasing a level of understanding or whether it involves manipulating information to achieve a tangible task.. A number of these dimensions have dichotomous modes, and thus require separate documentary mechanisms to capture information in an efficient manner. It is this area in which many previous works falter; to create an accurate representation of activities in their entirety, the focus must not be on any single mode of design activity, but rather on creating combined approaches which cover all eventualities. The dichotomy of interest in this paper lies with synchronous and asynchronous working, where engineers may work as part of a group or as individuals and where different forms of record are necessary to adequately capture the processes and rationale employed in each mode.

\section{INTRODUCTION}

Undertaking design activities invariably results in the generation of a significant quantity of information, not only on 


\section{SYNCHRONOUS AND ASYNCHRONOUS MODES OF WORKING}

When undertaking a large collaborative engineering design project, the effective use and understanding of both synchronous and asynchronous modes of working becomes critical to the success of the project. This is also true of smaller, co-located projects for during these projects both modes of working will also be utilized. In engineering design, working synchronously involves individual designers and/or team members working on the same activity at the same time. Activities such as design related meetings are prime examples of social and collaborative instances where valuable information and knowledge can be communicated through channels such as speech, body language and even gesturing, which can be extremely difficult to both recognize and record. In many cases subsequent decisions can be linked to this sometimes tacit or implicit knowledge and information, therefore much of the rationale behind these decisions are lost.

When working asynchronously, an engineer focuses individually on a given problem. The specific approach that they may take, the methods that they use and the assumptions that influence their course of action are not generally recorded, and the electronic files that they may manipulate do not expressly indicate the processes followed or where supporting information was obtained. As such, it is difficult to revisit previous design activities either to reuse in similar situations or to reassess and potentially update the design activity in the light of new understanding.

With the ubiquity of computational tools much of the information that an engineer will manipulate during the course of their working will be computationally explicit, and by seeking to capture the manipulations performed on the information and the interdependencies between different information resources, it becomes possible to build up a view of the processes followed in asynchronous working.

In order to provide an accurate depiction of the activities performed in the two modes of working, two separate documentary mechanisms are proposed. These will be discussed separately, and means of coordinating them will be proposed. A case study will then be covered to expand on this coordination.

\subsection{SYNCHRONOUS MODES OF WORKING}

In the past decade, there has been a significant amount of work focused on aiding the capture of information within synchronous situations. It is widely acknowledged that social and collaborative instances such as meetings can provide significant advantage to organizations later in the product lifecycle if they are properly documented. However, this body of work has taken either one of two approaches, the record everything and analyse later method or the analyse and document at the time method. The latter of these approaches tends to focus on techniques such as Issue-Based Information Systems (IBIS) documenting the decisions made and the issues resolved using structured argumentations. However, this approach does not record much of the dialogue which goes along with decisions and therefore valuable information can be lost. On the other hand, effort has been focused on developing systems which simply record everything using video and audio etc. but this approach results in masses of information being captured, some of which can be regarded simply as noise, and as such the post processing time is extensive.

The key issue as the authors sees it is the need to bridge the gap between current 'live capture' and 'post processing' systems by utilizing a combination of principles from both. A key aspect of previous work on 'live capture' is the structuring of the information in a way which can be easily visualized and followed by the users, as it occurs [6]. This is the area in which most IBIS based tools excel [7, 8]. The structuring of information according to the argumentation effectively provides a 'road map' to the exchanges, allowing users to follow the development process and understand how the design evolved to the final state, including the right and wrong turns which the discussion may have taken throughout. However, the real-time display of this information throughout an actual meeting can also help guide the discussion in the meeting and therefore IBIS tools are much more than simply documentation tools, they can in fact be considered as tools which aid the actual development process and can influence the direction in which the process goes.

The framework proposed within this paper will utilize timelines to create a recognizable structure for the users to follow the information flow. The metadata which can be extracted from various elements of multimodal information such as audio, video, word processor documents images etc. can provide a defined and easily traceable structure which can be displayed within software systems. In addition, the ability to upload and view media such as video and audio associated with the meeting at a later date enhances the overall record of the meeting and draws on the principle employed by systems focused on 'post processing' where the key factor is that a complete and accurate record of the meeting can be captured using video and audio. The key difference in this work is that using a set of guiding principles, users will have greater knowledge of the critical information exchanges to capture and therefore avoid capturing everything and subsequently reduce the work load. The key goal of this work is to enhance the record of activities and decisions made collaboratively and in social situations to allow them to be revisited at later stages in the design process, but without creating additional work for the participants

To this end, work has been performed on developing the Media Enhanced Minuting System (MEMS), a prototype software system to be used as an effective meeting capture and review tool. MEMS possesses the ability to quickly and unobtrusively create richer representations of meetings by displaying and organizing multi-media generated during the meeting. Using pre-defined meeting structure templates and drag and drop file upload facilities, the user has the ability to record not only the decisions and actions specified during 
meetings, but also to document the rationale generation and exploration activities which in effect led to the decisions and actions being taken.

\subsubsection{MEDIA ENHANCED MINUTING SYSTEM (MEMS)}

The MEMS tool itself is web-based software linked to a repository, allowing the user to easily and rapidly create a record of the meeting at the time as opposed to creating digital or paper-based minutes retrospectively. Essentially the software has a three layered structure, an underlying relational database, hypertext preprocessor (PHP) scripts to interrogate the database and a Flash ${ }^{\mathrm{TM}}$-based user interface (UI) to input, retrieve and display objects using the PHP scripts as the data translator.

Data objects such as images, video, audio and documents are uploaded to the database using the Flash-based "drag and drop" upload form and metadata such as time, date, meeting identifier, agenda item etc. are automatically associated with the data, relative to the specific point in the meeting at which upload occurs. The PHP scripts convert the object URLs into $\mathrm{xml}$ format, allowing the UI to display the data object links as data "blocks" on the meeting timeline. This approach allows users to quickly and easily visualize what objects were presented or used at any given point in the meeting, and as the blocks are directly linked to the database, user's can simply click on the block within the timeline and the relative data file is returned. The MEMS interface also possesses the ability to display the data objects in individual "data streams" customizable to specific user's requirements (figure 1).

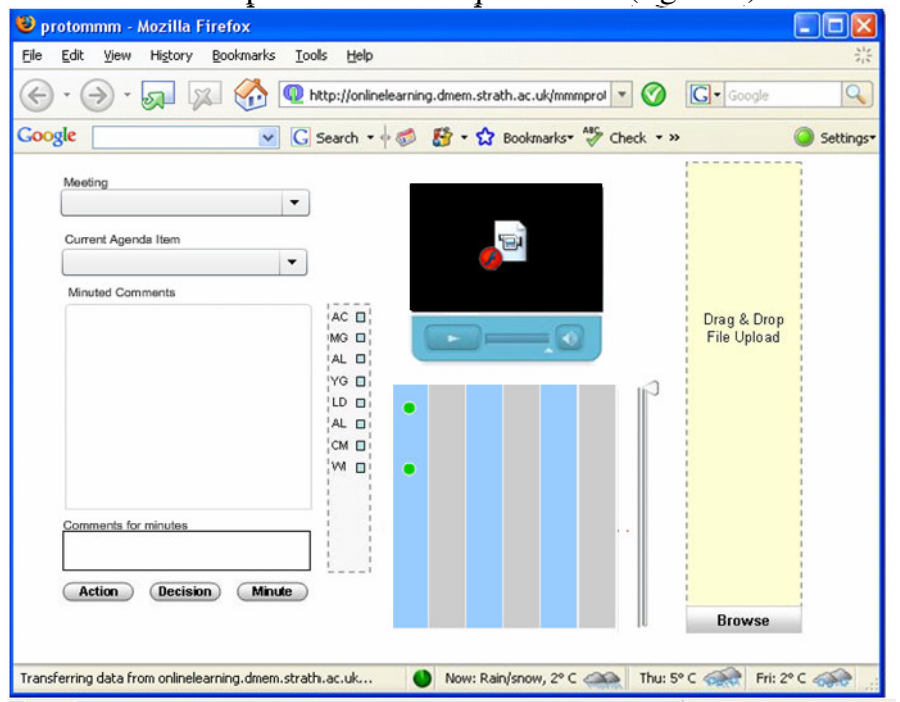

Figure 1: MEMS User Interface

Throughout the meeting, MEMS displays the currently active agenda item and metadata for the meeting (time, date, attendees) and the use of the dynamic database and PHP scripts allows a real-time view of the meeting to be viewed against time. Post-meeting, media streams such as video and audio are incorporated into the system and synchronized using the meeting timeline. These additional data streams, which are also displayed against the agenda points, are accessible and allow the user to revisit any part of the meeting record and view the data objects corresponding to a specific instance or occurrence during the meeting.

MEMS differs from previous work as it tries to bridge the gap between current live capture and post processing systems by utilizing a combination of both principles. The users have the ability to see what is being utilized in the meeting and the use of timelines allow an easily recognizable structure to be developed for the meeting, drawing from the principle of IBIS and the work done by Conklin [7] and Bracewell et al. [8] to name but a few. The ability to view all media associated with the meeting at a later date enhances the overall record of the meeting and draws on the principle employed by systems such as Informedia [9] and the Ferret browser [2] whose key selling point is that a richer representation of meeting activities can be captured using video and audio. The key goal of the MEMS development is to enhance the record of activities and decisions made collaboratively and in social situations to allow them to be revisited at latter stages in the design process, whilst also supporting the need to document real-time synchronous activities.

\subsection{ASYNCHRONOUS MODES OF WORKING}

The MEMS system essentially strives to provide a record of discursive or argumentative activities both by capturing viewable video or audio clips of a specific discussion and by recording the external information resources which were used to support or otherwise inform such argumentation. As such, the record provides a description of how information was manipulated within a discursive activity.

The asynchronous record shares this ambition, seeking to identify where external information is utilised within a specific activity and the mechanisms by which is manipulated or otherwise utilised to generate further information. Where the reliant upon cognitive processes, those within an asynchronous activity may be more explicit, for example when conducting some form of analysis within a computer-based environment whose manipulations are explicitly encoded. If it is possible to capture how these explicit manipulations are deployed and the information resources under manipulation, it becomes possible to build, from the ground-up, a record of the processes followed within an asynchronous activity.

In general, this record of detailed process is rarely captured in detail in existing documentary practices. Current documentary practice has been identified as sub-optimal, failing to capture activities and decisions in their entirety. There are other approaches which consider design processes from a modelling perspective. Such approaches include those based on Petri-nets [10], Decision Structure Matrices [11] and other more business-oriented process modelling approaches such as Business Process Modelling [12]. In general, these tend to be implemented at a high level, where they are well-suited to partitioning the design process into distinct areas of activity and indicating some form of precedence. These approaches are not 
generally applied at a detailed level, and hence do not capture the manipulations of information conducted by an individual engineer.

It is argued that generating a literal, low-level record of synchronous activities allows for greater re-use of previous design work, both in terms of the activities necessary to develop a design through to completion but also in what considerations were addressed and what specific information guided this development process. It becomes possible to revisit a previous design, both to apply the design process to a new problem (if sufficient context of the design is understood and it can be seen to be applicable in a new application) and to update the existing design to take account of new understanding of any aspect of the design, for example of new analytical methods, improved materials, customer requirements etc.

In addition to the distinction between synchronous and asynchronous activities, we consider a second distinction (that applies especially in asynchronous working) between "transaction" and "learning" activities. In transaction activities some specific information manipulation is carried out to produce new information, and the relationship between this input and output information and the manipulation mechanism may be recorded explicitly. The manipulation mechanism may be used repeatedly over multiple transformations. A learning activity, by contrast, is carried out to produce new knowledge or understanding which may be built upon by subsequent activities addressing the same issue. Each type of activity requires a different recording mechanism. In this paper we concentrate on records of transaction activities. We are working on approaches to making records of learning activities through the use of WiKis and other collaborative approaches such as social bookmarking. These approaches will be the subject of a separate paper.

\subsubsection{FORM OF TRANSACTIONAL RECORDS}

The approach taken in this research is to formally document the transaction activities conducted in asynchronous working at the level of the individual computational method, and then assemble these into a network of activities based upon information dependencies. It does not seek to create an automatically re-executable design record, although that remains a possibility in certain applications; instead, it is intended to assist the engineer in comprehending previous design activities. Although in principle a number of different representations may be applied, in this work the IDEF0 format [IEEE, 13] has been selected as the basis of the data model. As such, the record comprises a reference to activity, the information inputs and outputs, the resources (mechanisms) deployed in the analysis and the controls enacted upon the activity. Further supporting elements are the author (the engineer who created the record) and the start and end dates, together with text descriptions that allow, for example, assumptions to be recorded. It is possible to incorporate any further data element that may be deemed necessary.
A software environment has been developed which allows for the capture and recording of transactional activities, the automated compilation of reports from these records, and a means of browsing across the information interdependencies to visualise the 'audit trail' of a design episode. These three aspects will be briefly discussed in turn in sections 2.2.3 to 2.2.5.

\subsubsection{TRANSFORMATION OF UNDERPINNING RECORDS}

A fundamental principle in the presentation and organization of the records is that of transformation, where the generated reports are transformations of the underpinning activity records. This allows a single record (or set of records) to be utilized for multiple reporting purposes. This builds on the approach used in the Chemical Markup Language ChemML (and other related areas), where a given set of XML records can be manipulated in various ways by different publishers to produce notably different derived representations of the original information for their particular audience [14]. A key element of such approaches is the use of XML as the data model, primarily as it is computer-interpretable and thus may be manipulated for different purposes. In the work reported here, the use of XML allows for activity records to be presented to an end user in a number of forms, and providing the information is held in wellformed XML records with a consistent set of elements and attributes, the form in which the information is presented may be held as a template for application to any information resources of interest. It also becomes possible to interface with other technologies for the organization and visualization of the information. A separate strand of research is looking into the application of faceted classification to engineering design information [15], for which the ability to parse XML files for automated classification is essential, however in this work the application of Topic Maps as a visual interface is under exploration. Of the various different Topic Map syntaxes, XTM [16] is XML-based and thus can interface with the fundamental XML records of activity. This is discussed in section 2.2.4

\subsubsection{THE CAPTURE OF TRANSACTIONS}

In order to capture the manipulations of information within a transactional activity it is essential that 'atoms' of information may be identified and computationally addressed. In many Computer-Aided Engineering (CAE) environments it is possible to define objects which represent the various items of information necessary for conducting an analysis according to a pre-defined method or means of manipulation.

This approach is being explored with a large engineering company, that is radically overhauling its structural analysis approaches to centre on a shared CAE environment and associated PDM-based data and information repository. The initial work successfully introduced the notions of faceted classification to the storage of information objects within the repository [for a coverage of faceted classification please see 17]. The second round of work focuses upon capturing how the 
objects held within the repository are utilized within a given analysis, such that activity reports may be automatically generated. This will be extended to investigate how high-level reporting documents, as held in a separate repository, may be generated from these records.

In the research environment, a client-based tool has been developed to produce an XML representation of a transactional activity via the use of a form-based user interface. This tool allows all information and resources utilized in an activity to be referenced, and the resultant record captured using IDEF0 notation in an XML document. Related work on automatically capturing the activities performed within an immersive Virtual Reality environment [18], conducted under the auspices of the same broader research project, is being explored with a view to automatically capturing records of transactional design work, however work looking into how this may be carried out remains in its infancy.

\subsubsection{AGGREGATION AND PRESENTATION OF RECORDS}

The construction of an XML record of activity allows any number of further manipulations to transform the information as desired. The use of eXtensible Stylesheet Language Transformations [XSLT, 19] allows underpinning information represented in XML format to be algorithmically transformed into a given view, for example extracting data from a range of underpinning files to populate a table in a webpage. Using this technique, any number of 'report templates' can be constructed for purposes of displaying the underpinning information in a manner most suitable for a given reporting task, for example for communicating to a manager the resources utilised within a period of design work or for communicating to an engineer the final outputs of a previous episode of detailed design. It is possible to take values from a number of different XML files for this purpose, and the ability to reference distinct elements within each record allows only a specific item of information to be identified (for example a specific input, the source of the information, the author etc.) allowing a composite report which may aggregate information from multiple records to be constructed. XSLT templates have for example been constructed to display activities in a variety of tabular forms [20], an example of which may be seen in section 4.1.

\subsubsection{THE BROWSING OF AUDIT TRAILS}

The XSLT reports may be used to present information pertaining to a specific set of activities; however a separate method is needed to locate particular activities of interest from a set. It is also important, in terms of re-use, to allow an engineer to browse across a previous design record via its information dependencies to locate items or activities of interest. As such, a means of visualising the flow of information throughout the design activity is considered necessary.

Topic Maps are considered a suitable technology for this requirement. A Topic Map, in simple terms, comprises three distinct elements: topics, associations and occurrences [21] The topics represent concepts within the underlying records, for example in the present application a topic may be a given piece of information used as an input, a method, an activity etc. and the associations depict how these topics are inter-related and what role each topic plays in that specific relationship. This mapping exists as an abstract layer above the records, and occurrences indicate via URI where within the record the specific topic may be located.

The nature of the topics and their associations are specified within the activity records, including a title of the information entity, what other entities it is related to (in terms of IDEF0 linkages) and the electronic location of the entity itself. As these records are in XML syntax, it is possible to identify and transform these records into a syntax amenable to presentation as a Topic Map. Two key standards exist for Topic Maps, ISO 13250 [British Standards, 22] and XTM [16]. The consortium behind the XTM standard identified XML as a key enabling technology; hence this work uses the XTM syntax to express Topic Maps. A JavaScript has been created to automatically create a Topic Map record for each activity record, as the software used in development (Ontopia Omnigator) requires a specific Topic Map file to be located at a specific electronic location to enable processing.

Topic Maps also allow automated construction of larger Maps from smaller chunks. This facility, merging, allows a Topic Map transformation of a single activity to be combined into a complete map. The unique identity of a given topic can be specified by referencing a Published Subject Indicator [PSI, 23] essentially indicating where the definitive occurrence of that topic may be located, and when two topics in separate maps share the same PSI they are combined into a single topic upon merging. The associations of each topic are then assigned to this single topic.

It is possible to visualise this mapping, as it forms a nodearc diagram that may be traversed according to any given association of interest. An example of such a visualised map is given in section 5

\section{CO-ORDINATING THE REPRESENTATIONS}

The documentation of information within both synchronous and asynchronous modes of working allows for some degree of reuse of transactional work and for some degree of comprehension of the reasons behind decisions made in synchronous working. Individually however, they do not provide a complete depiction of the processes across the complete design episode. For example, the rationale leading to a transactional activity being undertaken may be exchanged in a synchronous episode, or the basis for a decision made within a synchronous mode of working may have been established in an asynchronous transaction. To enable these relationships to be recorded this it is essential that records from the two modes be co-ordinated such that dependencies can be traced. The means by which this will be accomplished is still an open area of 
research, and this section will discuss the avenues currently under consideration.

It is suggested that through the capture of the transition between synchronous and asynchronous working it becomes possible to provide a mechanism to group the transactional work into a single task. By capturing the instigation and completion of a task, at the points at which it interfaces with the synchronous modes, a natural partition is created. This partition clusters the contained transactions into a consistent set of activities, helping to indicate the broader purpose of a group of such transactions. The distinct transactions may be considered as progressive stages carried out to complete a wider task, and thus the boundaries around these activities must be identifiable to provide some context to the work.

In our observations of design activities we see transactional activities leading to synchronous meetings, and vice versa - e.g. meetings called to address conflicts or issues raised within transactional working or transactional activities instigated to address issues raised in synchronous working. Both modes of co-ordination may be pertinent, depending upon which direction of transfer is significant at a given point in the design process.

\subsection{INFORMATION DEPENDENCY CAPTURE}

It has been argued that by tracing the information flow within asynchronous activities it is possible to revisit such episodes. Extending the information dependency trail into the synchronous mode it is possible to capture how asynchronous activities impact upon decisions made within the synchronous environment. Two key related issues are faced, one of granularity and one of identification of key information pertinent to decision-making. Transactional records capture information flows at a lower level than within synchronous modes, where the emphasis is more upon aggregated information which is used in the identification and resolution of issues and in the support of decision-making. In many cases the aggregated information may comprise reports, presentations or other devices intended for purely communicative purposes, which is based upon the underpinning information utilized and developed within the transactional activities but not expressly linked to it. These boundary or intermediary objects are key to information exchange in such situations, and by utilizing XSLT transformations it is possible to express transactional records in such a manner as to support decision-making in synchronous situations. Different stakeholders within synchronous activities may have different viewpoints and thus need different transformations; however as the transformations are rigorously linked to the underpinning transactional record it is possible to comprehend the flow of information from transaction into synchronous modes of work. The MEMS system also makes reference to documents used in the support of discourse; hence linking synchronous events to the underpinning documentary resource.

\subsection{THE SPECIFICATION OF AGENDA}

It is possible to treat synchronous modes as setting the agenda for further work, whereby the transactional (and indeed learning) activities to be performed are specified or otherwise guided by the outputs of a synchronous meeting. This allows the decisions made within the synchronous to be linked to subsequent transaction activities. For example, if a part or component within a subassembly was found to be suboptimal then this part would have to be withdrawn or altered. The decision to undertake this redesign exercise would commonly be taken in collaborative situations such as design reviews or design change meetings and therefore fall under the synchronous modes of working.

However, the actual directive or action for the activity to be undertaken would in effect provide a structure or plan for the individual engineer and therefore the dependencies become visible. The key decisions and actions documented during the synchronous activities have the potential to be utilized as directives within process maps, informing the project stakeholders of the need to undertake certain activities.

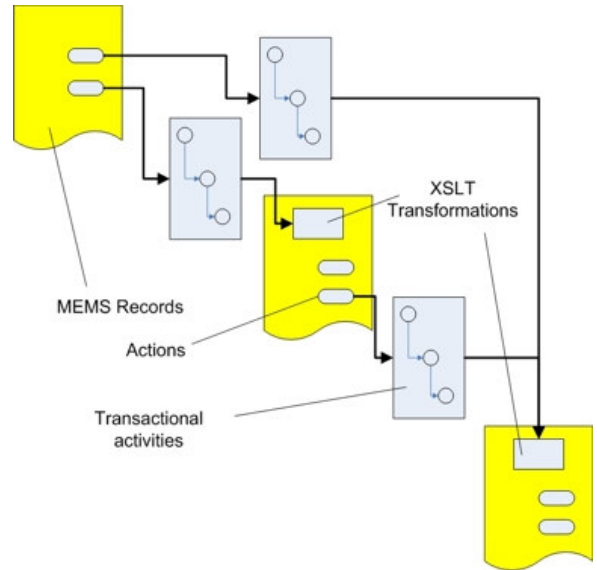

Figure 2: Co-ordination between MEMS and Transactional record

The representation of the activities may be mapped using for example IDEF models, showing the dependencies and the resources required during the synchronous activities. Figure 2 illustrates the means by which the two different forms of record may be co-ordinated, with actions from MEMS records setting the agenda for transactional activities, and XSLT views of transactional activities being referenced and utilized in synchronous activities.

\section{CASE STUDY}

In order to demonstrate the specific aspects of both the MEMS and transactional records and of how they may be combined to create a richer record of design, a simple case study will be presented. 


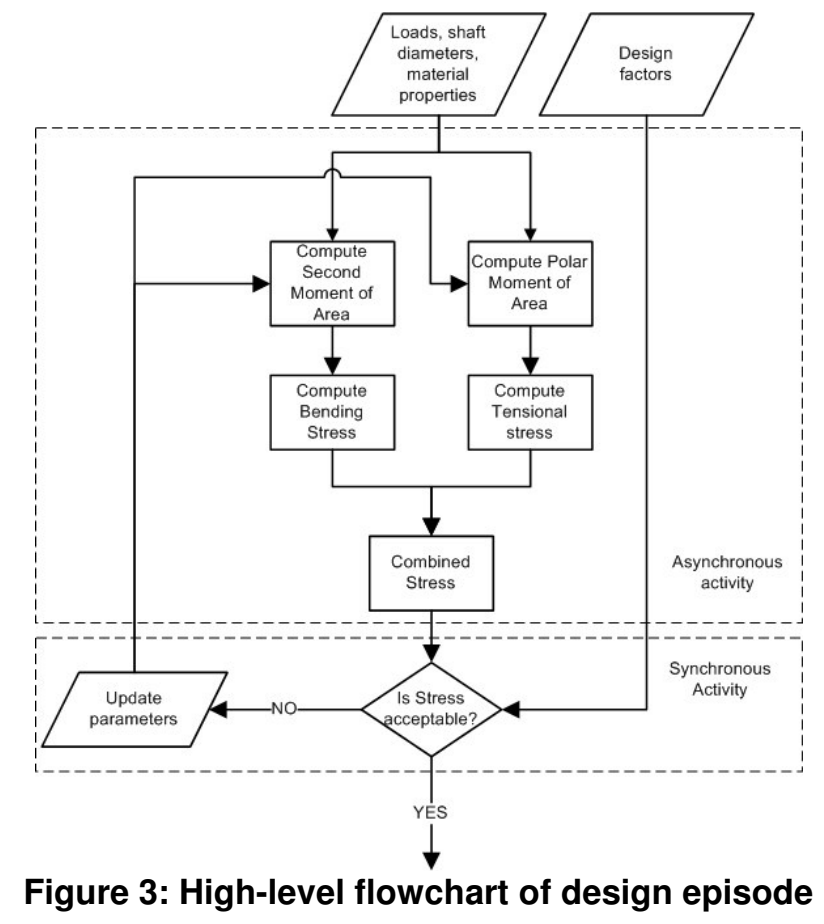

This activity was conducted by researchers within the Innovative Design and Manufacturing Research Centre (IdMRC) at the University of Bath as a means of validating and demonstrating the documentary approaches described in this paper. Figure 3 shows the high-level plan for the episode of design that is of interest in this paper. This is part of a broader case study, where the embodiment and detail stages of a snowmobile drive shaft design have been conducted for the purposes of testing the documentary approaches described in this paper. For reasons of brevity, only a small section of this design will be discussed in this paper.

This episode features a series of transactions, where computational analysis of the shaft under previously computed loads leads to a value of torsional and bending stresses, which may then be translated to a combined stress. At this point, a synchronous activity is undertaken where these stresses are considered in light of material properties and design factors (again previously computed) such that a decision may be made to reconsider any aspect of the design should it be subjected to excessive stress. At this point, the previous transactional activities may be repeated to ensure that the updated design meets the structural requirements.

The design activity was conducted using Matlab as the analytical engine for each transactional activity, and the method used was stored as a separate information resource which could be applied to identical tasks performed at other points in the design process. The various parameters and instances of information generated during each activity were stored in an XML file such that later activities could refer directly to the information of interest. A script was written to convert the information entities into appropriate format for use in the Matlab methods.
Upon encountering a synchronous activity, the information required for these activities was presented in its native format. The XSLT templates necessary to present this information in a more readily comprehensible manner were identified and retrospectively created. It is anticipated that by creating a library of such templates it would be possible to present future transactional records to engineers engaged in a synchronous activity in a convenient manner.

\subsection{RECORD OF TRANSACTIONAL ACTIVITIES}

Figure 4 shows the XSLT report generated from the underpinning records for the transactional activities. This particular report provides details of the information inputs and outputs to a particular activity, where the values for each input are hyperlinked to the underpinning XML records such that an engineer may retrieve and re-use this information if necessary. A description of the method is also given; this is included within the representation of the methods itself, such that an engineer may understand the nature of the manipulation applied to the information.

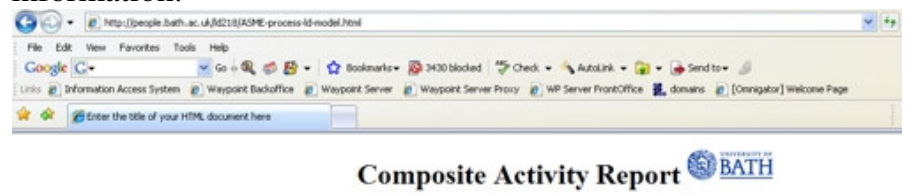

10.-6ne-

Please select a data set to transform:

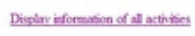

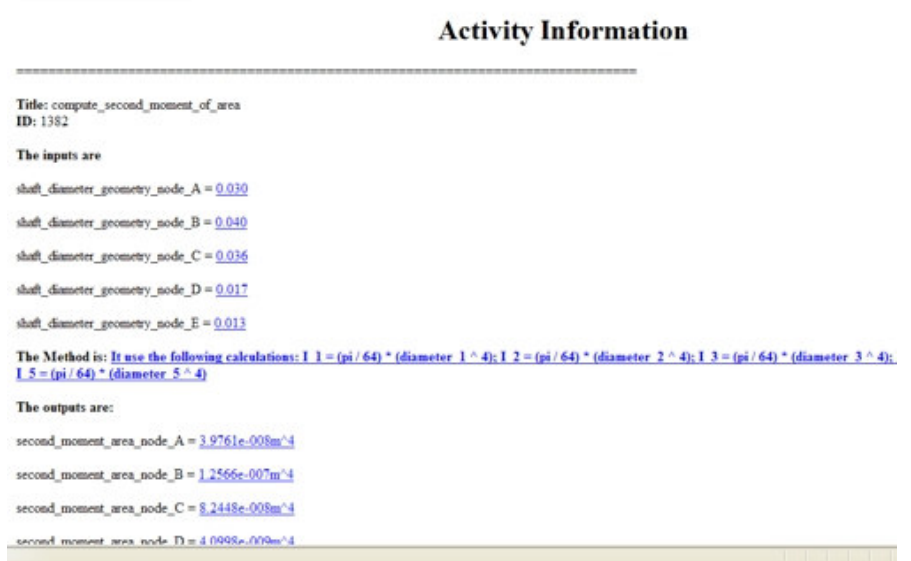

Figure 4: Case study XSLT report

Although not utilized in the 'live' experiment, this particular XSLT report contains information which would be useful to the engineer when attempting to reduce peak stresses, as it indicates that the value of second moment of area is proportional to the 4th power of shaft diameter, meaning that only slight increases to shaft diameter are necessary to cause significant increases to the moment of area. This became important when considering how to reduce peak stresses, and once an increase in diameter was identified as necessary an informed estimate as to the size of increase could be made. 


\subsection{RECORD OF SYNCHRONOUS ACTIVITIES}

As highlighted previously, multimodal records of synchronous activities can be generated using the MEMS software. The synchronous activity presented for the purposes of this paper considered the stress values calculated during transactional activities with respect to material properties and design factors, allowing an informed decision to be reached on the validity of the design. Within this activity, previously generated documentation such as spreadsheets, calculations, and XSLT reports were used as evidence in the discussions leading to the decision. The use of MEMS to document this activity allowed these data sources to be uploaded in real-time using the Flash-based drag and drop upload form at the point in the discussion at which they were utilized.

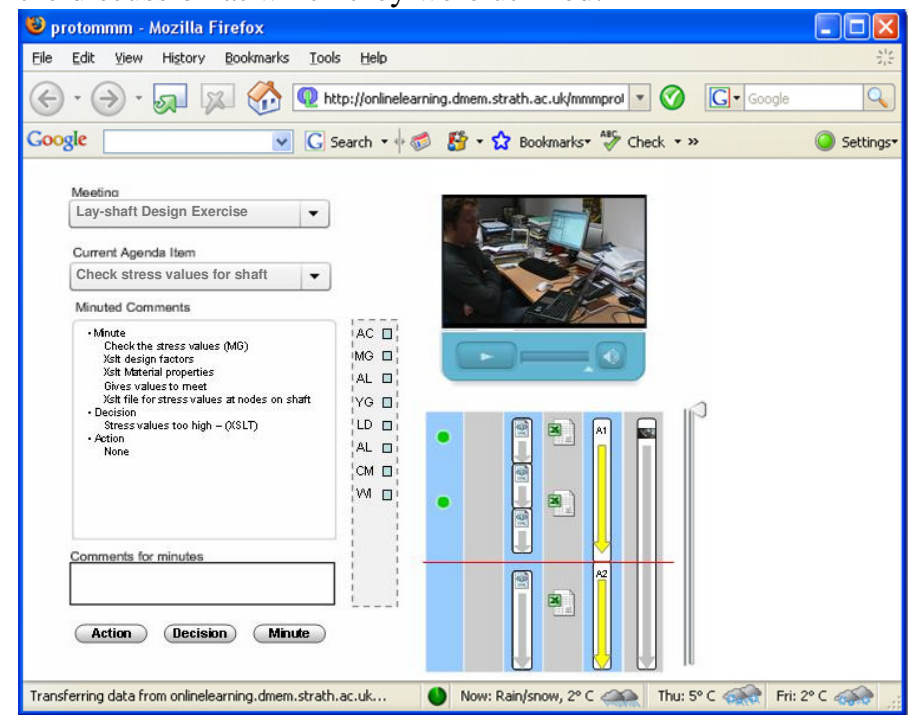

Figure 5: Case study MEMS record

As is shown in figure 5, this allowed the individual data sources such as the XSLT reports to be recorded and synchronized with the meeting timeline and the audio, video and text records created during the activity, returning an accurate and richer representation of the discussions and the point in time at which each element was presented.

\subsection{THE COORDINATED REPRESENTATION}

Fig 6 (Annex A) shows the complete Topic Map for the first iteration of the design episode, where the final outcome is the updated shaft geometry, modified as the shaft stresses were seen to be excessive and a decision was taken to reconsider the shaft geometry to address this. The nodes of the Topic Map are informed by the IDEF0 approach, with information resources represented as topics which flow into and out of activity topics, with the resources located beneath the activity to which they are associated. This map can be dynamically updated to suit any perspective, for example it is possible to view all the activities into which an information resource acts as input to, or to view where a given method has been employed.

\section{CONCLUSIONS}

The work introduced in this paper has served to highlight the possibility of creating richer and more accurate records of design activities. This paper introduced complimentary approaches to achieving representations of design activities performed synchronously and asynchronously, and by doing so has highlighted the benefits of each approach. The Media Enhanced Minuting System (MEMS) was developed specifically to capture the discursive and collaborative aspects of synchronous design activities, and in doing so, documents not only the discussions and decisions made therein, but also the information resources utilized within such design situations. Transactional records are utilized in capturing the information manipulations in asynchronous working, and allow various transformations to be applied to present the information dependencies within these activities in a variety of different forms according to user need. These two forms of documentation may then be coordinated by considering how the use of transactional record transforms within synchronous modes may be captured, and how actions from synchronous events may act as an 'agenda' for transactional activities in the asynchronous mode. Through the undertaking of a design based case study, the records generated by each approach serve to highlight the possibility of providing a more complete depiction of activities undertaken, and in doing so, provide positive direction for future co-development of such documentary activities.

\section{ACKNOWLEDGEMENTS}

The work presented herein was undertaken under the aegis of a larger investigation on knowledge and information management funded primarily by the Engineering and Physical Sciences Research Council (EPSRC) and the Economic and Social Research Council (ESRC). The "Knowledge and Information Management (KIM) Through-Life Grand Challenge Project" [24] brings together a team of some 70 academics and researchers from 11 UK based universities to look at the knowledge management challenges associated with a move towards through-life product support.

\section{REFERENCES}

1. Conway, A.P., et al., A Study of Information and Knowledge Generated During Engineering Design Meetings, in International Conference on Engineering Design (ICED). 2007: Paris, France.

2. Lalanne, D., et al., The IM2 Multimodal Meeting Browser Family, in Joint IM2 Technical Report. 2005.

3. Huet, G., Design Transaction Monitoring: Understanding Design Reviews For Extended Knowledge Capture, in Mechanical Engineering. 2006, University of Bath. p. 298. 
4. Moran, T., et al., "I'll Get That Off the Audio": A Case Study of Salvaging Multimedia Meeting Records, in CHI'97. 1997: Atlanta, GA, USA.

5. Wiil, U.K., Proceedings of the 5.5 Open Hypermedia System Working Group Meeting, U.K. Wiil, Editor. 1999, Aalborg University: Esbjerg.

6. Conklin, J., Dialog Mapping: Reflections on an Industrial Strength Case Study, in Visualising argumentation:software tools for collaborative and educational sense-making, P.A. Kirschner, S.J. Buckingham-Shum, and C.S. Carr, Editors. 2003, Springer-Verlag: London.

7. Conklin, J., Dialogue Mapping: Building Shared Understanding of Wicked Problems. 2006, Chichester: John Wiley \& Sons, Ltd.

8. Bracewell, R.H., S. Ahmed, and K.M. Wallace, DRed and design folders: a way of capturing, storing and passing on knowledge generated during design projects, in ASME International Design Engineering Technical Conferences \& Computers and Information in Engineering Conference. 2004: Salt Lake City, Utah, USA,.

9. Hauptmann, A., et al. Video Retrieval with the Informedia Digital Video Library System. in Text Retrieval Conference (TREC'01). 2001. Gaithersburg, Maryland.

10. McMahon, C. and X. Meng, A Network Appraoch to Parametric Design Integration. Research in engineering Design, 1996. 8: p. 14-32.

11. Steward, D.V., The design structure system - A method for managing the design of complex systems. IEEE Transactions on Engineering Management, 1981. EM28: p. 71-74.

12. White, S.A. Introduction to BPMN. 2002 [cited 2008 15 Jan 2008]; Available from: http://www.bpmn.org/Documents/Introduction\%20to\% 20BPMN.pdf.

13. Systems, K.B., IEEE Standard for Functional Modeling Language - Syntax and Semantics for IDEFO. 1998.

14. Murray-Rust, P. and H.S. Rzepa, Scientific Publications in XML - Towards a Global Knowledge Base. Data Science, 2002. 1: p. 84-98.

15. Giess, M.D. and C.A. McMahon. The Application of Faceted Classification in the support of Manufacturing Process Selection. in Decision strategies in design: an interdisciplinary approach. 2007. Delft Centre for Materials TU Delft, The Netherlands.

16. TopicMaps.Org. XML Topic Maps (XTM) 1.0. 2001 [cited 20061 April 2006]; Available from: http://www.topicmaps.org/xtm/.

17. Giess, M.D., P.J. Wild, and C.A. McMahon, The Generation Of Faceted Classification Schemes For Use In The Organisation Of Engineering Design
Documents. International Journal of Information Management, 2008((accepted for publication)).

18. Rea, H., et al. Automating Digital Capture of Engineering Information. in Proceedings of the 4th International CIRP-Sponsored Conference (DET2007) Digital Enterprise Technology. 2007. University of Bath, UK.

19. Clark, J. XSL Transformations (XSLT) Version 1.0. 1999 [cited 20071 December 2007]; Available from: http://www.w3.org/TR/xslt.

20. Ding, L., et al., Component-based Model: a Process Model for Designers. (In Preparation).

21. Pepper, S. The TAO of Topic Maps. 2002 [cited 1 August 2006]; 19]. Available from: http://www.ontopia.net/topicmaps/materials/tao.html.

22. Information technology - SGML applications - topic maps. 2000.

23. Vatant, B., et al., Published Subjects: Introduction and Basic Requirements - OASIS Published Subjects Technical Committee Recommendation, 2003-06-24. 2003, OASIS.

24. McMahon, $\mathrm{C}$., et al. Knowledge and Information Management (KIM) Grand Challenge Project. 2006 [cited 2007 15th April]; Available from: http://wwwedc.eng.cam.ac.uk/kim/. 
ANNEX A

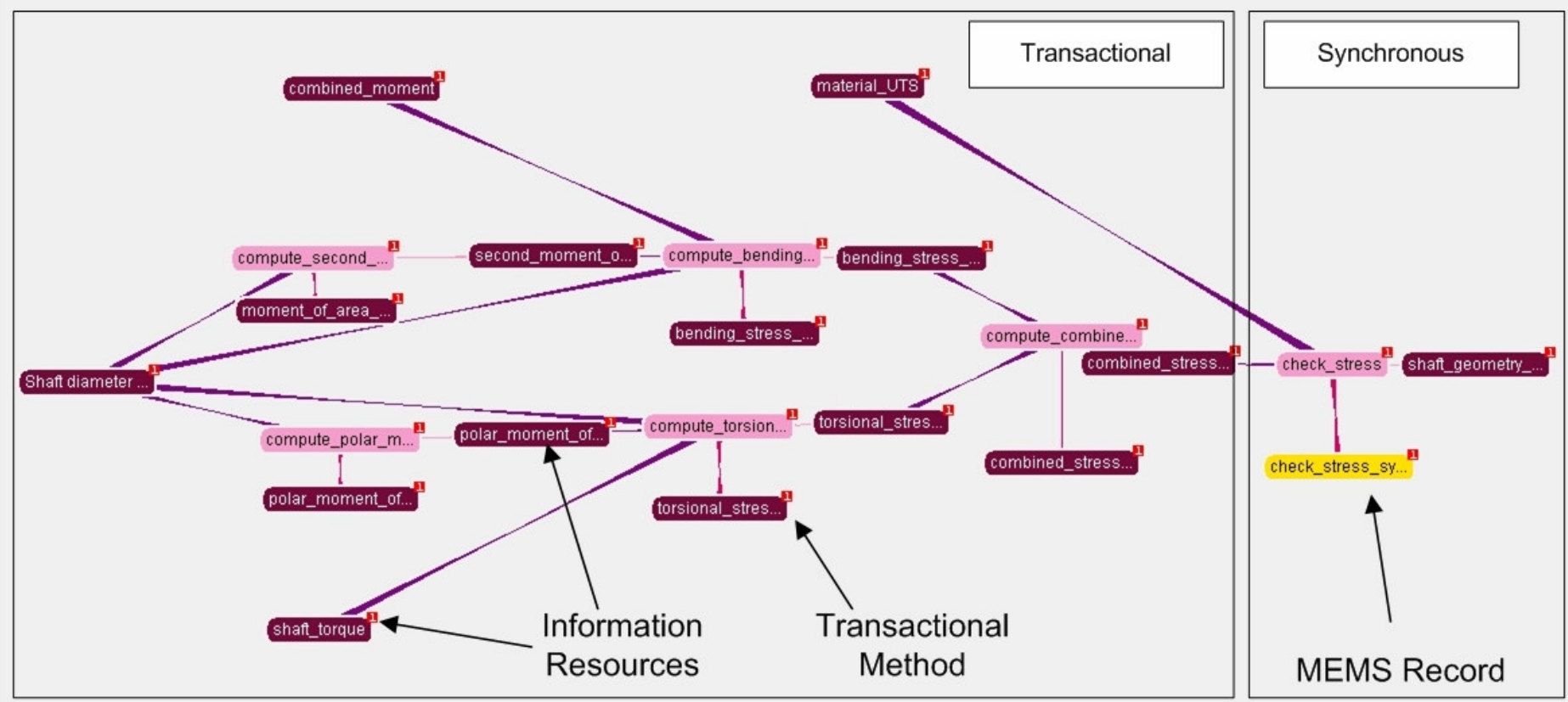

Figure 6: Sample Topic Map of Transactional Episode 